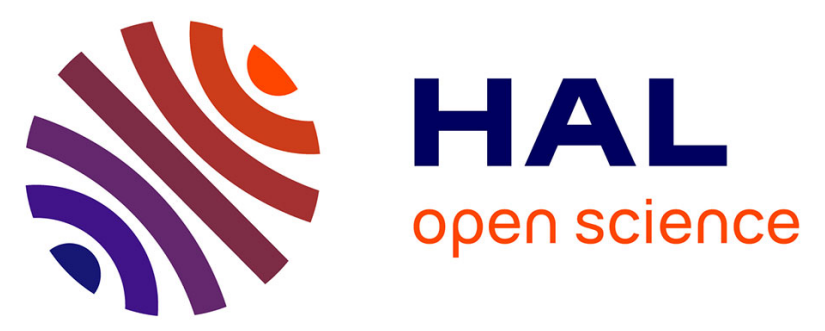

\title{
Performance and Energy Aware Wavelength Allocation on Ring-Based WDM 3D Optical NoC
}

Jiating Luo, A Elantably, D D Pham, C Killian, Daniel Chillet, Sébastien Le Beux, Olivier Sentieys, Ian O'Connor

\section{- To cite this version:}

Jiating Luo, A Elantably, D D Pham, C Killian, Daniel Chillet, et al.. Performance and Energy Aware Wavelength Allocation on Ring-Based WDM 3D Optical NoC. Design, Automation \& Test in Europe Conference \& Exhibition (DATE 2017), Mar 2017, Lausanne, Switzerland. hal-01416958

\section{HAL Id: hal-01416958 \\ https://hal.inria.fr/hal-01416958}

Submitted on 15 Dec 2016

HAL is a multi-disciplinary open access archive for the deposit and dissemination of scientific research documents, whether they are published or not. The documents may come from teaching and research institutions in France or abroad, or from public or private research centers.
L'archive ouverte pluridisciplinaire HAL, est destinée au dépôt et à la diffusion de documents scientifiques de niveau recherche, publiés ou non, émanant des établissements d'enseignement et de recherche français ou étrangers, des laboratoires publics ou privés. 


\title{
Performance and Energy Aware Wavelength Allocation on Ring-Based WDM 3D Optical NoC
}

\author{
J.Luo*, A.Elantably*, V.D.Pham*, C.Killian*, D.Chillet* $^{*}$ \\ S.Le Beux ${ }^{\dagger}$, O.Sentieys* and I. O'Connor ${ }^{\dagger}$ \\ *University of Rennes 1, Lannion, 22300 France, firstname.lastname@irisa.fr \\ ${ }^{\dagger}$ Ecole Centrale de Lyon, INL, Ecully, F-69134, France, firstname.lastname@ec-lyon.fr
}

\begin{abstract}
Optical Network-on-Chip (ONoC) is a promising communication medium for large-scale Multiprocessor System on Chip (MPSoC). ONoC outperforms classical electrical NoC in terms of throughput and latency. The medium can support multiple transactions at the same time on different wavelengths by using Wavelength Division Multiplexing (WDM). Moreover multiple wavelengths can be used as high-bandwidth channel to reduce transmission time. However, multiple signals sharing simultaneously a waveguide can lead to inter-channel crosstalk noise. This problem impacts the Signal to Noise Ratio (SNR) of the optical signal, which leads to an increase in the Bit Error Rate (BER) at the receiver side. In this paper we first formulate the crosstalk noise and execution time models and then propose a Wavelength Allocation (WA) method in a ring-based WDM ONoC allowing to search for performance and energy trade-offs, based on the application constraints. As result, most promising WA solutions are highlighted for a defined application mapping onto 16-core WDM ONoC.
\end{abstract}

\section{INTRODUCTION}

Evolution of Multiprocessor System-on-Chip (MPSoC) is moving towards the integration of hundreds of cores on a single chip. Designing such complex MPSoC is very challenging. In particular, designing the communication media is one of the most critical part of such systems. A large number of classical Network-on-Chips (NoC) were designed and proposed in the literature. However, these NoCs suffer from some limitations due to the electrical interconnect characteristics: capacitive and inductive coupling [1], interconnect noise and propagation delay. Thus, a new on-chip interconnect technology that can overcome these limitations is highly desirable.

Recent progresses in silicon photonics device manufacturing allow to rely on Optical Network-on-Chip (ONoC) for on-chip MPSoC communications. ONoCs are based on waveguides, carrying optical signals, and optical devices allowing to inject or drop the signals into this waveguide from an electrical interface. This technology offers low latency and high bandwidth properties. The waveguide for payload transmission can be shared by multiple senders and receivers when WDM [2] is used to support multiple transactions simultaneously in a shared waveguide, providing a significant increase in bandwidth efficiency. Indeed, several communications between different couples of source and destination cores can be established in parallel by using different wavelengths. However, simultaneous transmissions, on close adjacent wavelengths, may introduce inter-channel crosstalk noise through different optical switching elements within the network [3]. This inter-channel crosstalk leads to an increase of the laser power when a specific Bit Error Rate (BER) is targeted. Figure 2 shows how crosstalk is generated when multiple wavelengths pass through an ON or OFF state Micro Resonators (MR). A MR is an optical filter dedicated to a resonant wavelength that depends on the material and the design choices. Nevertheless, MRs are not ideal and drop also an amount of power of others wavelengths. This undesirable optical power is inversely proportional to the distance between the resonance wavelength and the optical signal wavelength.

When an application runs on the system and uses ONoC to ensure the communications between processors, two fundamentally sources of crosstalk noise appears: inter-communication and intra-communication crosstalk. The first one occurs when two different transmissions share the same waveguide simultaneously. The second one depends on the effect of undesirable coupling between different wavelengths used for the same transmission. When the task mapping allows to move the communication in space and in time respectively, the first type of crosstalk may be limited. However, the second type of crosstalk will always be there until the communication finishes. Moreover, the amount of crosstalk noise is largely dependent on the number of co-propagating wavelengths.

Increased bandwidth parallelism can reduce transmission time, but it results in a higher crosstalk. High bandwidth utilization and BER performance are thus two conflicting objectives. In order to get the best performance/energy tradeoff, this paper addresses, for the first time, the multi-objective problem of Wavelength Allocation (WA) for ring-based WDM ONoC. This work introduces a multi-optimization model and then proposes an approach based on the Non-dominated Sorting Genetic Algorithm II (NSGA-II) [4], to get a set of Paretooptimal solutions. The solution considers application-dependant transmission between source and destination, which is critical for performance optimization. The ring-based ONoC model is genetic so that one can parameterize the total number of wavelengths. The exploration is made by searching WA solution minimizing global execution time, BER and bit energy. The main contribution of this paper is a wavelength allocation exploration for ring-based $\mathrm{ONoC}$ architecture. The Pareto solutions give the most promising WA for performance/energy trade off.

This paper is organized as follows. Section II presents the related works. Section III presents the architecture, power loss models, time models and the wavelength allocation methodol- 
ogy using genetic algorithm. Then, Section IV presents some results produced by our approach. Finally, Section V concludes this paper and presents some perspectives.

\section{RELATED WORK}

The WA problem for classical optical network is addressed by several works. Zang et al. have resumed the assignment approaches for WDM optical network [5]. WA can be classified into two types: dynamic time and static time. In dynamic time, the lightpath is set-up on demand. In static time, lightpath requests are known in advance, and the wavelength assignment operation is performed off-line. Some heuristic algorithms have been proposed [6], including Random Wavelength Assignment, First-Fit, Most-Used and Least-Used algorithms. The majority of these approaches aim to reduce the overall blocking probability for new connections.

WA for ONoC shares some different characteristics with classical optical networks. For example, the distinctive properties of photonics component (i.e MR acts as a filter) cause some problems, including undesirable mode coupling between adjacent wavelengths in the photodetector that generate crosstalk noise and impact potentially the Optical NoC [7]. Therefore, the WA methodology specific to ONoC should be studied.

The wavelength routed based interconnection solutions such as RPNoC [8] and ORNoC [9] employ a design time WA approach. Then, they do not require any arbitration to reserve an optical path before data transmission. However, the allocation is fully static and the connection are fixed for one architecture even if different applications are executed.

Research about the crosstalk effect in ONoC was also conducted. Nikdast et al. developed models at the device level for the worst-case and average crosstalk noise in different ONoCs [10]. However, reducing the worst-case crosstalk noise is not sufficient if targeting a performance/energy trade-off for a specific application. Recently, a mapping tool has been released and contributes to reduce the crosstalk by reducing the number of communications sharing a waveguide [11]. It can avoid intercommunication crosstalk by moving task in space and time. However, this tool performs the analyses of power loss and crosstalk noise for single-wavelength ONoCs. Chittamuru et al. propose a crosstalk mitigation technique to increase channel spacing between adjacent wavelengths in dense wavelength division multiplexing (DWDM) [7]. However, they improve SNR performance of system by reducing DWDM degree, the WA issue is not considered in this work.

To the best of our knowledge, none of previous work explored WA for WDM ONoC. In this paper, we propose a genetic approach to solve the multi-objective WA problem.

\section{Multi-ObJective Optimization}

In this section, we firstly present the WDM-based ONoC ring which is considered in this paper. Then, we present the power loss model and the time model used for system optimization. Finally, the WA methodology is explained.

\section{A. Considered ONoC architecture based on WDM}

The model of optical loss of an ONoC not only depends on the network topology but also on the communication model. In this paper, we focus on the ring-based architectures and the WDM communication protocol.

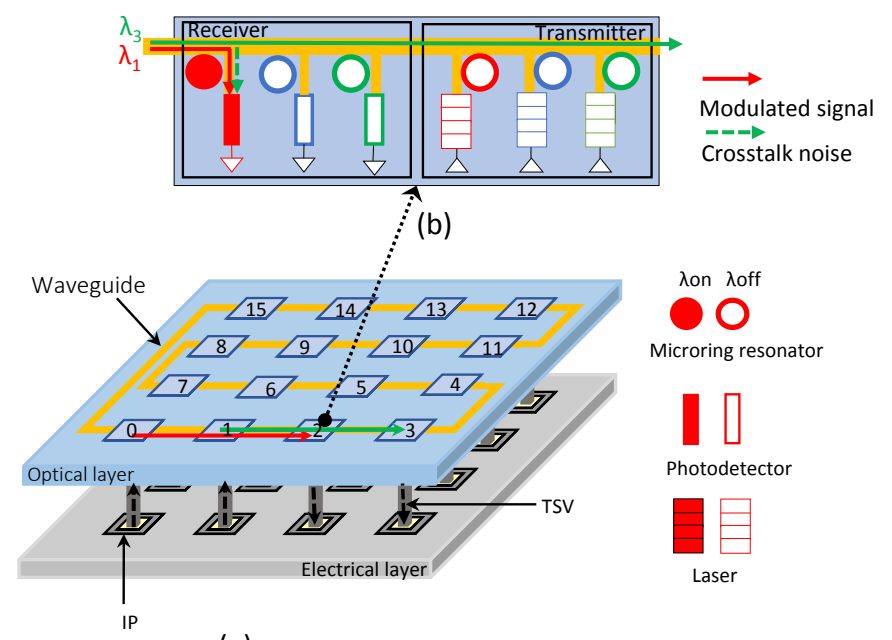

(a)

Fig. 1: Architecture Overview: a) 3D Optical Manycore ; b) Optical Network Interface

Figure 1.a shows how a ring-based ONoC architecture is defined. It is composed of two layers: i) an electrical layer implementing $n \times n(4 \times 4$ in Figure 1.a) IP cores and ii) an optical layer integrating the ONoC.To connect IP cores to the waveguide, an Optical Network Interface (ONI) is implemented for each IP core (see Figure 1.b). Each ONI is crossed by a waveguide propagating optical signals and integrates a receiver and a transmitter. The waveguide implements a WDM technique with $N W$ wavelengths. The receiver part is made of wavelengthspecific MR that can be turned ON or OFF to configure drop (receive) or pass-through operations of the signals at a corresponding wavelength, respectively. The transmitter is composed of on-chip laser sources that can emit and inject optical signal at a specific wavelength into the waveguide. The data are directly transmitted from these lasers through current modulation (OOK modulation).

\section{B. Power loss model}

Optical integrated waveguides have been already studied, and several publications deal with the losses of each optical elements. In [12], authors demonstrate that the loss $\Phi$ induced by a MR is linked to the distance between the resonance frequency of the MR $\lambda_{m}$ and the frequency of a wavelength $\lambda_{i} . \Phi$ is defined by

$$
\Phi\left(\lambda_{i}, \lambda_{m}\right)=\frac{P_{d r o p}^{\lambda_{i}}}{P_{\text {input }}^{\lambda_{i}}}=\frac{\delta^{2}}{\left(\lambda_{i}-\lambda_{m}\right)^{2}+\delta^{2}}
$$

where $2 \delta$ is the $-3 d B$ bandwidth of the MR filter, which can be defined as $Q=\frac{\lambda_{m}}{2 \delta}$, with $\mathrm{Q}$ the quality factor of the MR. $P_{\text {input }}^{\lambda_{i}}$ is the optical power at input port. $P_{d r o p}^{\lambda_{i}}$ is the power at the 
drop port. From Eq. 1, it is clear that the choice of wavelengths for communications sharing the same part of a waveguide has a direct impact on the noise introduced at the photodetector input. In this paper, we suppose equal Channel Spacing (CS) between two consecutive wavelengths covering a whole Free Spectral Range (FSR). For our context, we focus on the receiver part, where the MRs are used to extract an optical signal from the main waveguide to drive them toward the photodetector.

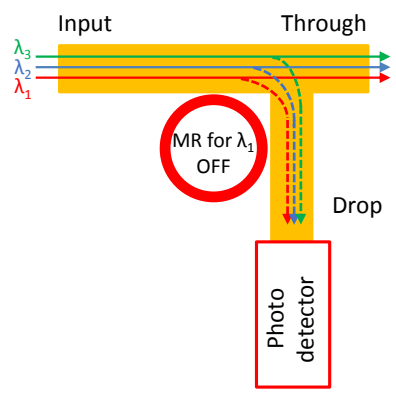

(a)

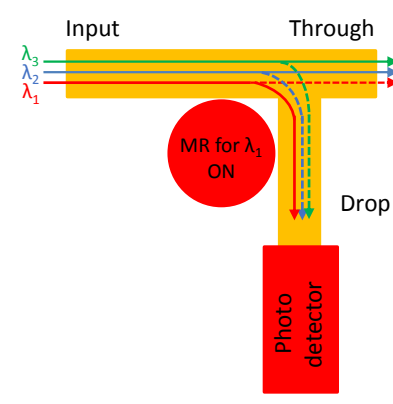

(b)
Fig. 2: Analysis of signal dropping by a MR element for a) MR in OFF-state; b) MR in ON-state

Figure 2 shows OFF- and ON-state of a MR placed along the waveguide. In the OFF-state of a MR (Fig. 2.a), all the optical wavelengths travel towards the through port to the waveguide, the signal power $P_{T, M o f f} f_{\lambda_{m}}[i]$ of $\lambda_{i}$ at the through port of OFFstate $\mathrm{MR}_{\lambda_{m}}$ is

$$
\begin{aligned}
P_{T, M o f f}[i]= & P_{\text {input }}[i]+L_{p 0} \\
& \forall i \in\{1, \ldots, N W\}
\end{aligned}
$$

with $L_{p 0}$ the power loss in OFF-state of MR, $P_{\text {input }}[i]$ the power of optical signal $\lambda_{i}$ at input port of the $\mathrm{MR}_{\lambda_{m}}$. The power $P_{D, M o f} f_{\lambda_{m}}[i]$ of $\lambda_{i}$ at drop port is

$$
\begin{aligned}
& P_{D, M o f f_{\lambda_{m}}}[i]= \begin{cases}P_{\text {input }}[i]+K_{p 0} & i=m \\
P_{\text {input }}[i]+\Phi_{d B}\left(\lambda_{m}, \lambda_{i}\right) & i \neq m\end{cases} \\
& \forall i \in\{1, \ldots, N W\}
\end{aligned}
$$

with $K_{p 0}$ the crosstalk coefficient of OFF-state MR. $\Phi\left(\lambda_{m}, \lambda_{i}\right)$ calculates the filtered power of other wavelengths.

When the MR at $\lambda_{m}$ is ON-state, the impact is different. As shown in Figure 2.b, the optical signal on $\lambda_{1}$ is filtered by the ON-state MR specific to $\lambda_{1}$. Meanwhile, other signals travel toward the through port, but a portion of power of these signals is leaked to the drop port, introducing inter-channel crosstalk noise on the detected signal expressed by $\Phi\left(\lambda_{i}, \lambda_{m}\right)$. Therefore, the power $P_{T, \text { Mon }_{\lambda_{m}}}[i]$ of $\lambda_{i}$ at the through port is

$$
\begin{aligned}
P_{T, \text { Mon }_{\lambda_{m}}}[i]= & \left\{\begin{array}{cc}
P_{\text {input }}[i]+L_{p 1} & i \neq m \\
P_{\text {input }}[i]+K_{p 1} & i=m
\end{array}\right. \\
& \forall i \in\{1, \ldots, N W\}
\end{aligned}
$$

with $L_{p 1}$ the power loss in ON-state of $\mathrm{MR}$ and $K_{p 1}$ is the crosstalk coefficient of ON-state MR. The power $P_{D, M o n_{\lambda_{m}}}[i]$ of $\lambda_{i}$ at the drop port is

$$
\begin{aligned}
P_{D, \text { Mon }_{\lambda_{m}}}[i]= & \begin{cases}P_{\text {input }}[i]+L_{p 1} & i=m \\
P_{\text {input }}[i]+\Phi_{d B}\left(\lambda_{m}, \lambda_{i}\right) & i \neq m\end{cases} \\
& \forall i \in\{1, \ldots, N W\}
\end{aligned}
$$

Finally, from these previous analyses, Eq. 6 computes the signal power received at photodetector of $\lambda_{m}, P_{\lambda_{m}}^{\text {signal }}$, while Eq. 7 calculates the crosstalk noise from the optical signals on the other wavelengths received at the photodetector, $P_{\lambda_{m}}^{\text {noise }}$.

$$
\begin{aligned}
& P_{\lambda_{m}}^{\text {signal }}=P_{v}+L_{p 0}[m]+L_{p 1}[m]+L_{P}[m]+L_{B}[m] \\
& P_{\lambda_{m}}^{\text {noise }}=\sum_{i \neq m}^{M} P_{v}+L_{p 0}[i]+L_{p 1}[i]+L_{P}[i]+L_{B}[i]+\Phi_{d B}\left(\lambda_{m}, \lambda_{i}\right)
\end{aligned}
$$

where $M$ is the total number of other wavelengths passing through the photodector specific to $\lambda_{m} . P_{v}$ represents the on-chip Vertical Cavity Surface Emitting Laser (VCSEL) power. $L_{P}[i]$ and $L_{B}[i]$ are the propagation and bending losses of $\lambda_{i}$. The exact number of bendings and the distance travelled can be derivated from the architecture. $L_{p 0}[i]$ and $L_{p 1}[i]$ are the losses of OFFstate and $\mathrm{ON}$-state MR of $\lambda_{i}$ due to the various intermediate tiles along the path. Please note that we only consider the first-order inter-channel crosstalk noise in this paper.

It is important to notice that VCSEL is switched ON and OFF to represent data 1 and 0 . Ideally, no light should be emitted when zeros are transmitted [13], where $P^{1}=P_{\text {input }}$ and $P^{0}=0$ using $P_{\text {input }}$ to denote the input optical power of modulator. However, we shall notice that this is not always the case in practical implementation. Therefore, the non-zero power of 0 is also considered as part of the noise. A simplified SNR model for the input of the photodetector of $\lambda_{m}$ can be defined by

$$
S N R_{\lambda_{m}}=\frac{P_{\lambda_{m}}^{\text {signal }}}{P_{\lambda_{m}}^{\text {noise }}+P^{0}}
$$

Finally, the BER of direct detection with an OOK modulation [14] is expressed by Eq. 9. The crosstalk problem can be formulated as:

$$
\text { Minimize } \quad B E R=\frac{1}{2} e^{-\frac{S N R}{2}} \times\left(1+\frac{S N R}{4}\right)
$$

From the above analysis, we can see that the objective function Eq. 9 deeply depends on the number of co-propagating wavelengths.

\section{Time and application models}

Definition 1: A Task Graph $\mathrm{TG}=G(\mathcal{T}, \mathcal{D})$ is a directed graph, where each vertex $T_{i} \in \mathcal{T}$ represents one task. Each directed edge $d_{i, j}=\left(T_{i}, T_{j}\right) \in \mathcal{D}$ characterizes the communication from task $T_{i}$ to task $T_{j}$. The weight of edge is the communication volume exchanged by $T_{i}$ and $T_{j}$, denoted by $V\left(d_{i, j}\right)$.

Definition 2: An Architecture Characterization Graph ACG $=$ $G(P, L)$ is an undirected graph, where each vertex $p_{i} \in P$ represents one IP core in the architecture. Each edge $l_{i, j} \in L$ is a physical link connecting IP core $p_{i}$ and $p_{j}$. 
Definition 3: The mapping of TG onto the processors of ACG is defined by one-to-one mapping function $\operatorname{map}(\mathcal{T})$ :

$$
\begin{aligned}
\text { map }: \mathcal{T} \rightarrow P \Longrightarrow & p_{i}=\operatorname{map}\left(T_{i}\right) \\
& \forall\left(T_{i}, T_{j}\right) \in \mathcal{T}^{2} \exists\left(p_{i}, p_{j}\right) \in P^{2} \mid p_{i} \neq p_{j}
\end{aligned}
$$

The constraint above guarantees that each task in TG can be assigned to an IP core and ensures that different tasks are mapped on different IP cores.

Figure 3 illustrates the time chart of a given application task graph which has been mapped to an ONoC platform [15]. $T_{j}$ and $T_{k}$ are assigned to $p_{i}$ and $p_{m}$, respectively. We assume that each IP core is of the same type, so that the processing time of task is not relevant to the assigned IP. In order to formulate the time model, the following definitions are introduced:

- $t_{p}^{j}$ : the execution time of task $T_{j}$ in one processor

- $T_{j, k}$ : the communication time between $T_{j}$ and $T_{k}$

- $\operatorname{pre}\left(T_{j}\right)$ : the set of predecessors of task $T_{j}$

- $t_{\text {end }}^{j}$ : the completion time of task $T_{j}$

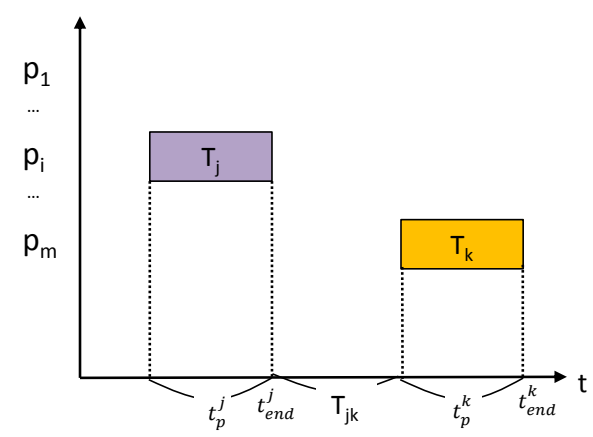

Fig. 3: Time chart of TG for $T_{j} \in \operatorname{pre}\left(T_{k}\right)$

The communication time between $T_{j}$ and $T_{k}$ can be expressed as

$$
T_{j, k}=\frac{V\left(d_{j, k}\right)}{N W_{j, k} \times B}
$$

where $B$ is the data rate per wavelength in the optical layer. $N W_{j, k}$ represents the number of reserved wavelengths for the communication between $T_{j}$ and $T_{k}$. The global execution time problem can be formulated as:

$$
\begin{array}{r}
\text { Minimize } \quad \text { Global execution time }=\operatorname{Max}\left(t_{\text {end }}^{k}\right) \forall k \in\left[1, N_{t}\right] \\
\text { with } t_{\text {end }}^{k}=t_{p}^{k}+\operatorname{Max}\left(t_{\text {end }}^{j}+T_{j, k}\right)_{\forall j \mid T_{j} \in \operatorname{pre}\left(T_{k}\right)}
\end{array}
$$

where $N_{t}$ is the total number of tasks in the TG. Multiple wavelengths for a given communication can open high bandwidth channels. This is suitable for scheduling communication time for each transaction to reach a high time efficiency.

\section{Wavelength Allocation Methodology}

The two models detailed in sections III-B and III-C show that the number of reserved wavelengths severely impacts BER and global execution time. As previously mentioned, a tradeoff between execution time and crosstalk noise must be found. The multi-objective wavelength allocation problem is thus NPhard and, in this case, heuristic approaches must be defined.
Evolutionary technique, such as genetic algorithm, is one of the most popular choice [4]. It exploits a fixed-sized population of candidate solutions (called chromosome) and evolves over a number of generations to reach a satisfactory solution [16].

$$
\left[c_{0} / \ldots / c_{k} / . . \cdots / c_{N_{l}-1}\right]
$$

(a)

\begin{tabular}{c|c}
\hline values & $W L$ \\
\hline $01 \cdots 0$ & $\lambda_{1} \lambda_{2} \cdots \lambda_{N W}$ \\
\hline
\end{tabular}

(b)
Fig. 4: (a) Chromosome structure (b) Corresponding values

Each solution (see Figure 4 for illustration) is represented by a binary array chromosome. The chromosome is composed of $N_{l} \times N W$ genes, where $N_{l}$ equals to the number of edges in TG. Every $N W$ genes specifies the wavelengths allocated for the communication $c_{k}$ (corresponding to one edge of TG with $\left.k \in\left[0, N_{l}-1\right]\right)$. Each gene specifies the state of wavelength (' 0 ': Not-reserved, '1': Reserved). For example, for a task graph with 6 communications (6 edges between tasks) and a waveguide supporting 4 wavelengths, the chromosome is defined by the model $[1000 / 0001 / 0001 / 0001 / 1000 / 1000]$. Furthermore, each $c_{k}$, defined by a value like [1000], specifies the wavelength allocation. For example, the first $c_{0}=[1000]$ represents the allocation of $\lambda_{1}$ to support the communication.

For the multi-objective wavelength allocation problem, the optimization process works as follows. Firstly, a random parent population $P_{0}$ of $N$ chromosomes is generated. Then the metrics can be obtained by the power and time models introduced in Section III. It is important to notice that the chromosome could be invalid in the following cases:

- Same wavelengths are assigned to the same link.

- The reserved wavelengths for one link exceed the bandwidth of waveguide.

If the generated chromosome is on one of these cases, we directly set the fitness to infinity. From each valid solution generated, we can then extract the solutions which ensure the trade-off between execution time and energy efficiency. Afterwards the mutation and crossover are employed to generate new population detailed as follows:

- Crossover: For two randomly chosen chromosomes, two random crossover points $x, y$ are selected. The two offsprings are generated by exchanging the genes $[x, y]$ of two parents.

- Mutation: A randomly point is chosen to be inverted.

Finally a Pareto-front can be reported for high-level decision when a set of communications must be managed.

\section{REsults}

To illustrate our approach and demonstrate the effect of WA on the performance, we study in this section an example based on a specific application mapping. The application chosen is presented in Figure 5. Based on this task graph, and for a ring-based ONoC architecture composed of $4 \times 4$ IP cores, we propose a mapping of tasks defined at design time.

The evolutionary algorithm is set to iterate 300 times with a population size of 400 individuals (individuals of the first 


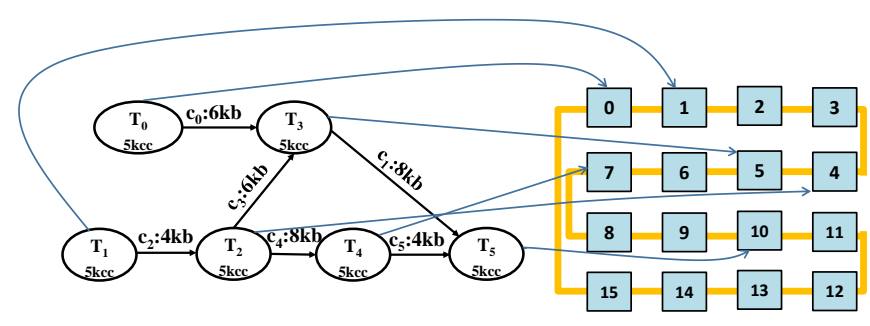

(a)

(b)

Fig. 5: a) Task graph of the virtual application; b) Mapping of the task graph onto the ring-based WDM ONoC architecture

iteration are randomly generated). For sake of simplicity, we discuss a set of Pareto solutions according to two objectives from $N W$ point of view. Each solution corresponds to a wavelength allocation result. We assume $P_{v}$ for transmission of 1 and 0 are $-10 \mathrm{dBm}$ and $-30 \mathrm{dBm}$, respectively. For the simulation, the MR is defined by FSR set to $12.8 \mathrm{~nm}$ and $Q$ set to 9600 . The other parameters, in particular power losses, are summarized in Table I. It should be noted that our methodology is independent of the values of parameters, which are only used as an example.

TABLE I: Power loss values

\begin{tabular}{|c|c|c|c|}
\hline Parameters & Symbol & Value & Ref \\
\hline Propagation loss & $L_{p}$ & $-0.274 \mathrm{~dB} / \mathrm{cm}$ & {$[17]$} \\
\hline Bending loss & $L_{b}$ & $-0.005 \mathrm{~dB} / 90^{\circ}$ & {$[18]$} \\
\hline Power loss: OFF-state MR & $L_{p 0}$ & $-0.005 \mathrm{~dB}$ & {$[18]$} \\
\hline Power loss: ON-state MR & $L_{p 1}$ & $-0.5 \mathrm{~dB}$ & {$[18]$} \\
\hline Crosstalk loss: OFF-state MR & $K_{p 0}$ & $-20 \mathrm{~dB}$ & {$[19]$} \\
\hline Crosstalk loss: ON-state MR & $K_{p 1}$ & $-25 \mathrm{~dB}$ & {$[19]$} \\
\hline
\end{tabular}

Figure 6(a) represents a set of Pareto solutions for different $N W(4,8$ and $12 \lambda)$ according to the global execution time and the bit energy. In order to explain the variation of execution time and simplify expressions, we illustrate, for some points of $12 \lambda$ WA solutions, the number of reserved wavelengths for each communication. For example, $[2,8,6,6,4,7]$ means that for communication $c_{0}, 2 \lambda$ are reserved, for $c_{1}, 8 \lambda$ are reserved and so on. We can observe that energy consumption per bit increases with the number of reserved wavelengths. This is due to the additional ON-state MRs suffering from more propagation loss in the architecture. Obviously, the solution with most energy saving is the allocation $[1,1,1,1,1,1]$ (i.e., one wavelength for each communication) ensuring the smallest number of parallel signals in the waveguide. If we consider $N W$ equals to 4 , the corresponding chromosome is $[1000 / 0001 / 0001 / 0001 / 1000 / 1000]$ as presented in section III-D. Moreover, the considerable improvement of execution time is achieved with growing number of reserved wavelengths.

As expected, the bit energy increases with $N W$. This is due to the additional optical components integrated in the ONoC and more propagation loss suffered by optical signal. However, the time efficiency is improved. Precisely, from 4 to 8 available $\lambda$, a significant improvement of optimized execution time is reached (here $28.3 k$-cc (clock cycles) for $4 \lambda$ and $23.8 k$-cc for $8 \lambda$ ). By comparing $8 \lambda$ and $12 \lambda$, only a slight improvement is obtained (here $23.8 k-c c$ for $8 \lambda$ and $22.96 k-c c$ for $12 \lambda$ ). Indeed, increasing the bandwidth reduce the communication time in the waveguide, hence reduce the global execution time of the application. This trend decreases when the communication time become negligible compared to the task execution time on the cores. Hence, the optimized execution time will tend to the minimal execution time ( $20 k-c c$ as shown in the figure), where task $T_{1}, T_{2}, T_{4}$ and $T_{5}$ are executed with a bandwidth so high that transmission time between tasks is negligible. It is worth mentioning that a growing number of wavelengths increases the area cost.

Figure 6(b) represents a set of Pareto solutions for different $N W(4,8$ and $12 \lambda)$ according to the global execution time and the average BER. As explained above, the array of the number of reserved wavelengths for $12 \lambda$ are displayed. From this figure, we can observe that the global execution time decreases with growing number of reserved wavelengths, overall. However, this improvement in execution time leads to a degradation of BER due to the increased parallel signals in the waveguide. From the $N W$ point of view, as $N W$ increases, the BER is nearly unchanged. This is due to the fixed FSR and defined tasks mapping. The BER result here is directly obtained from the calculation of SNR.

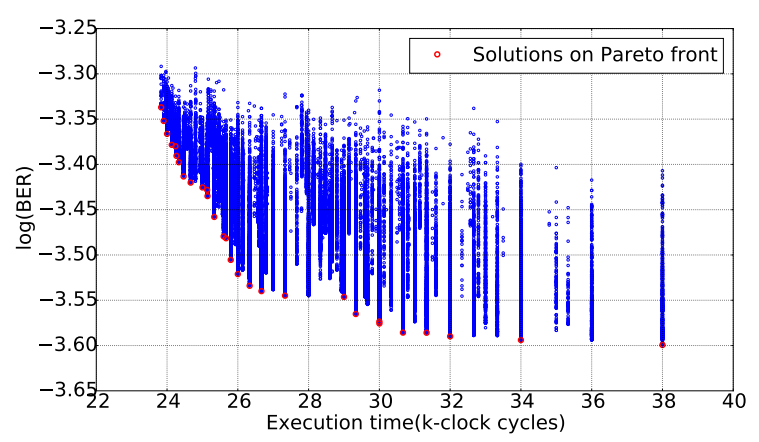

Fig. 7: Valid allocation solutions generated for 8 wavelengths

TABLE II: Amount of generated valid solutions and amount of solutions on the Pareto front for wavelength allocation

\begin{tabular}{|c|c|c|}
\hline $\begin{array}{c}\text { Number } \\
\text { of WL }\end{array}$ & $\begin{array}{c}\text { Number of solutions } \\
\text { on Pareto front }\end{array}$ & $\begin{array}{c}\text { Number of valid } \\
\text { solutions }\end{array}$ \\
\hline 4 & 10 & 28284 \\
\hline 8 & 29 & 86525 \\
\hline 12 & 51 & 100578 \\
\hline
\end{tabular}

In order to illustrate the complexity of wavelength allocation, Table II lists the number of valid solutions generated by the genetic algorithm and the number of solutions located on the Pareto front. For instance, for 8 wavelengths, and given the parameters of the genetic algorithm aforementioned, 86,525 valid solutions have been generated. Among them, only 29 solutions provide an efficient trade-off between energy and execution time. To illustrate theses results, figure 7 draws all the solution on a BER versus execution time plot for 8 wavelengths. We can note that a huge number of solution are far away from the Pareto front. This figure confirms that wavelength allocation must be carefully done in a ring $\mathrm{ONoC}$ otherwise leading to an inefficient use of the communication medium.

Our strategy focus on the wavelength allocation for an appli- 


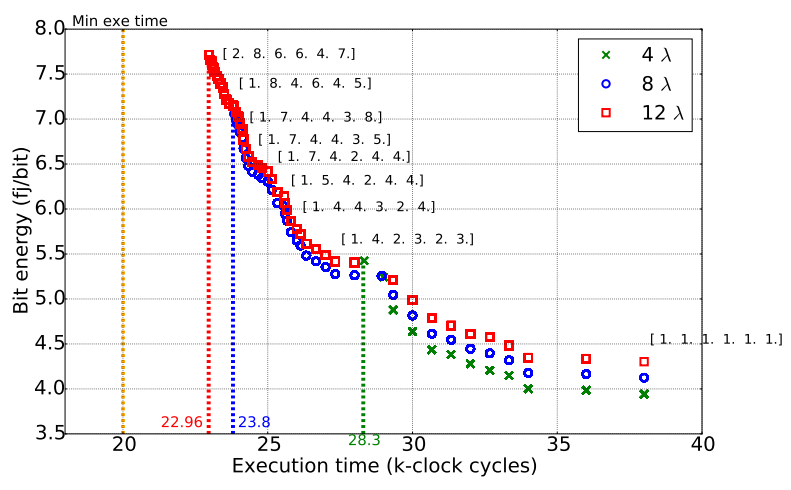

(a)

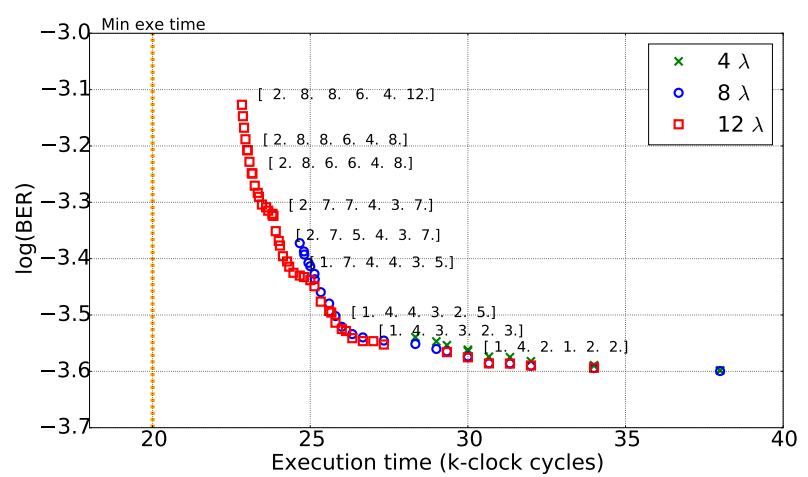

(b)

Fig. 6: Pareto solutions according to (a) bit energy versus global execution time (b) BER versus global execution time

cation whose mapping of tasks onto processors is known in advance. Except two general factors of genetic algorithm (size of population and generation), the complexity of our strategy is also driven by the size of application and the number of wavelengths (expressed as $\mathrm{O}\left(N_{l}^{2} N W^{2}\right)$ ).

\section{CONCLUSION}

Wavelength allocation is a critical issue for the BER and energy performance of WDM ONoC. In this paper, we demonstrate that allocation of multiple parallel optical signals to support communication on $\mathrm{ONoC}$ can improve the execution of task graph representing an application. However WDM introduces crosstalk between simultaneous communications located on the waveguide. This crosstalk depends on the distance between the optical signals which travel the waveguide at the same time. The consequence is a reduction of SNR of the communications depending on the wavelength selection. Therefore, we propose an approach enabling the concurrent optimization of WDM ONoC. A set of most promising solutions in a design space is obtained. As results, the most energy-efficient solution is obtained when each communication is performed on 1 wavelength. Moreover, the optimized time tends to the minimal execution time with growing number of wavelengths. From the designer point of view, trade-off is then possible to respect the application constraints in terms of energy or timing performance. Future work will concern the possibility to evaluate the performance for different task mapping. Since the task mapping allows to move the communication in space and in time respectively, the system performance including throughput, BER and bit energy will be better improved.

\section{REFERENCES}

[1] R. Ho et al., "The future of wires," Proceedings of the IEEE, vol. 89, no. 4, pp. 490-504, 2001.

[2] A. K. Dutta et al., WDM technologies: optical networks. Academic Press, 2004.

[3] L. H. K. Duong et al., "Coherent and incoherent crosstalk noise analyses in interchip/intrachip optical interconnection networks," IEEE Transactions on Very Large Scale Integration (VLSI) Systems, pp. 2475-2487, July 2016.
[4] K. Deb et al., "A fast elitist non-dominated sorting genetic algorithm for multi-objective optimization: Nsga-ii," in International Conference on Parallel Problem Solving From Nature. Springer, 2000, pp. 849-858.

[5] H. Zang et al., "A review of routing and wavelength assignment approaches for wavelength-routed optical wdm networks," Optical Networks Magazine, pp. 47-60, 2000.

[6] T. Thomas and K. Bala, "Multiwavelength optical networks: a layered approach," 1999.

[7] S.V.R. Chittamuru et al., "Improving crosstalk resilience with wavelength spacing in photonic crossbar-based network-on-chip architectures," in IEEE 58th International Midwest Symposium on Circuits and Systems (MWSCAS), 2015, pp. 1-4.

[8] X. Wang et al., "Rpnoc: a ring-based packet-switched optical network-onchip," IEEE Photonics Technology Letters, vol. 27, no. 4, pp. 423-426, 2015.

[9] S. Le Beux et al., "Optical ring network-on-chip (ornoc): Architecture and design methodology," in Design, Automation \& Test in Europe, 2011, pp. $1-6$.

[10] M. Nikdast et al., "Crosstalk noise in wdm-based optical networks-onchip: A formal study and comparison," IEEE Transactions on Very Large Scale Integration (VLSI) Systems, vol. 23, no. 11, pp. 2552-2565, 2015.

[11] E. Fusella et al., "Phonocmap: an application mapping tool for photonic networks-on-chip," in 2016 Design, Automation \& Test in Europe Conference \& Exhibition (DATE), 2016, pp. 289-292.

[12] S. Xiao et al., "Modeling and measurement of losses in silicon-oninsulator resonators and bends," Optics Express, vol. 15, no. 17, pp. 10 553-10 561, 2007.

[13] C. Peucheret, "Direct current modulation of semiconductor lasers," Lecture notes in "Introduction to Optical Communication", vol. 34140, pp. 1-7, 2006.

[14] M. Seimetz, High-order modulation for optical fiber transmission. Springer, 2009.

[15] R. Hwang et al., "A comparison of multiprocessor task scheduling algorithms with communication costs," Computers \& Operations Research, pp. 976-993, 2008.

[16] E. Fusella et al., "Crosstalk-aware mapping for tile-based optical networkon-chip," in High Performance Computing and Communications (HPCC). IEEE, 2015, pp. 1139-1142.

[17] P. Dong et al., "Low loss silicon waveguides for application of optical interconnects," in Proc. IEEE Photon. Soc. Summer Topical Meeting Ser, 2010, pp. 191-192.

[18] F. Xia et al., "Ultra-compact high order ring resonator filters using submicron silicon photonic wires for on-chip optical interconnects," Optics express, pp. 11 934-11941, 2007.

[19] J. Chan et al., "Physical-layer modeling and system-level design of chip-scale photonic interconnection networks," IEEE Transactions on computer-aided design of integrated circuits and systems, pp. 1507-1520, 2011. 